\title{
The World Health Organization dengue case classifications
}

\author{
Umesh Jayarajah $^{1}$, Upul Dissanayake ${ }^{2}$, Kamali Edirisinghe ${ }^{1}$, Suranjith L Seneviratne ${ }^{1,3}$ \\ ${ }^{\prime}$ Department of Surgery, Faculty of Medicine, University of Colombo, Sri Lanka. \\ ${ }^{2}$ Department of Medicine, National Hospital of Sri Lanka, Colombo, Sri Lanka. \\ ${ }^{3}$ Institute of Immunity and Transplantation, Royal Free Hospital and University College London, London, UK.
}

\author{
Correspondence: Prof. Suranjith L Seneviratne \\ e-mail:suran200@yahoo.co.uk \\ https://orcid.org/0000-0002-6548-5673
}

Dengue, a viral haemorrhagic fever causes a significant burden to healthcare systems of countries in the tropics and subtropics $(1,2)$. In recent years, considerable variations in disease presentations involving different organ systems have been documented (1, 3-7). The clinical importance of the different dengue case classifications has been a matter of debate $(8,9)$. The 1997 World Health Organization (WHO) dengue classification, classified the disease as: dengue fever (DF), dengue haemorrhagic fever (DHF), and dengue shock syndrome (DSS). In 2009, a revised classification was released which classified the disease into "dengue" and "severe dengue", with or without "warning signs" (Table 1) $(8,10)$.

Retrospective studies from Brazil and South-East Asia have compared the two WHO dengue case classification systems (Table 2 (11-16)). In 2011, Barniol, et al., included patients from the Eastern Mediterranean, American and South-East Asian regions and concluded the 2009 classification had greater clinical usefulness in dengue case management and surveillance (16). A large retrospective Brazilian study found considerable agreement between the two classification systems, but the 2009 guidelines had a higher sensitivity in the diagnoses of severe cases (11). Similar studies from Southeast Asia also found the 2009 classification to be more useful clinically $(14,15)$.

Studies from Sri Lanka have also compared the two dengue classification systems (Table 1). In 2017, Sri Lanka faced its largest dengue epidemic $(17,18)$. A large multicentre study (that included more than
1500 patients) from Sri Lanka during this epidemic compared the two WHO dengue classification systems (9). The 2009 dengue classification was found to be better at detecting disease severity. The number of warning signs correlated with the severity of dengue and the presence of four or more warnings signs was found to be significantly associated with severe dengue. A major drawback of the 1997 dengue classification is that it focuses mainly on plasma leakage and its associated haemodynamic instability, and fails to consider organ dysfunction as a manifestation of disease severity. For example, in the Jayarajah, et al., study, three DF patients were reclassified as severe dengue (according to the 2009 classification) due to the presence of organ dysfunction and excessive bleeding (9). Wanigasuriya, et al., and Bodinayake, et al., also found the 2009 dengue classification to be more useful $(19,20)$. Similarly, Jayaratne, et al., found the 2009 classification to be better and clinical findings such as abdominal pain, vomiting and bleeding manifestations were more prevalent in severe dengue (21).

Although the 2009 WHO dengue classification was more useful in diagnosing clinically severe disease, there are several practical concerns. According to the 2009 guidelines, only one warning sign is sufficient to alert physicians to the need for immediate assessment or intervention. Although, waiting for more than 3 warning signs to manifest would increase the predictive value, it may considerably delay diagnosis and intervention, and the patient may develop more severe disease with complications (9). 


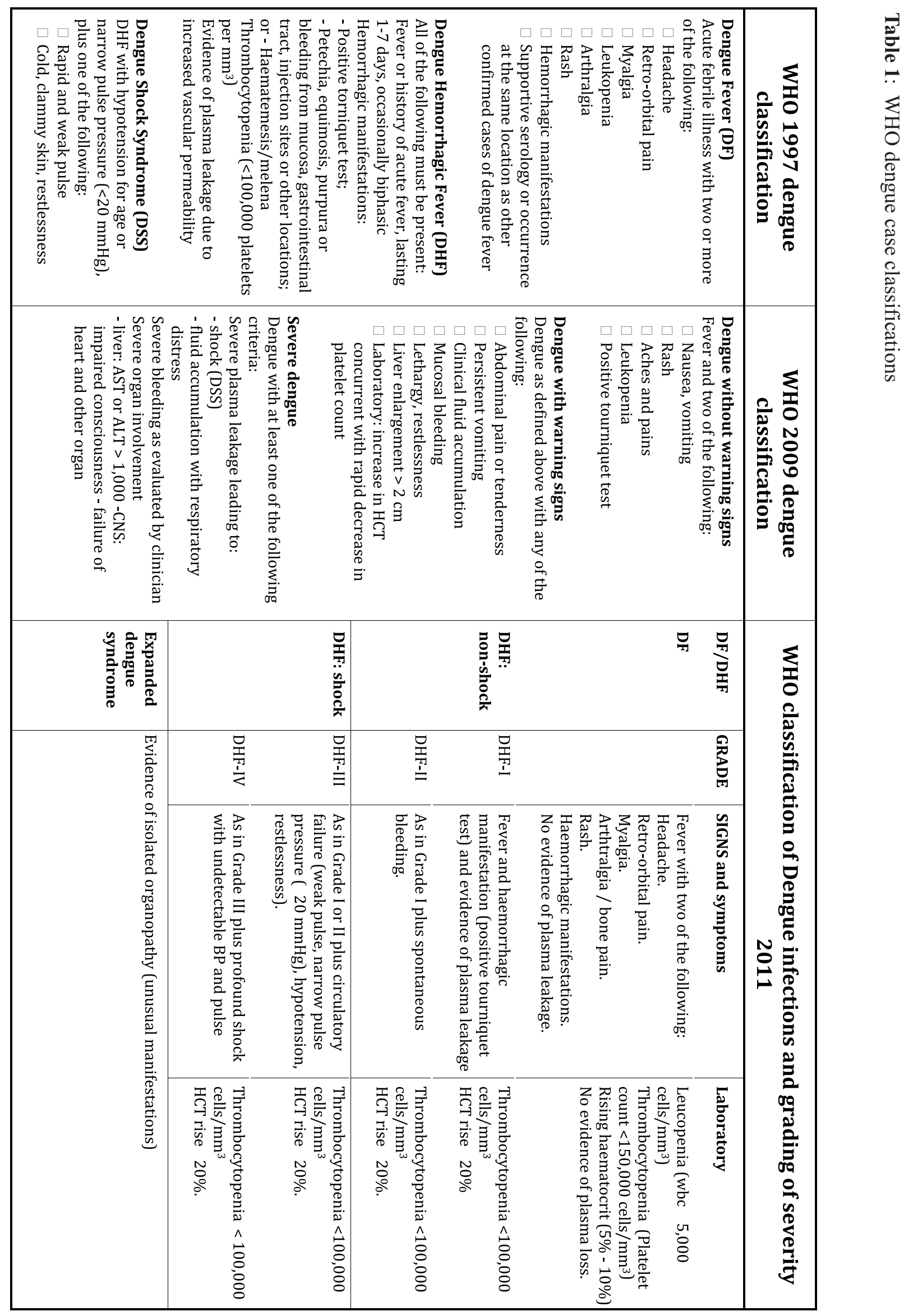




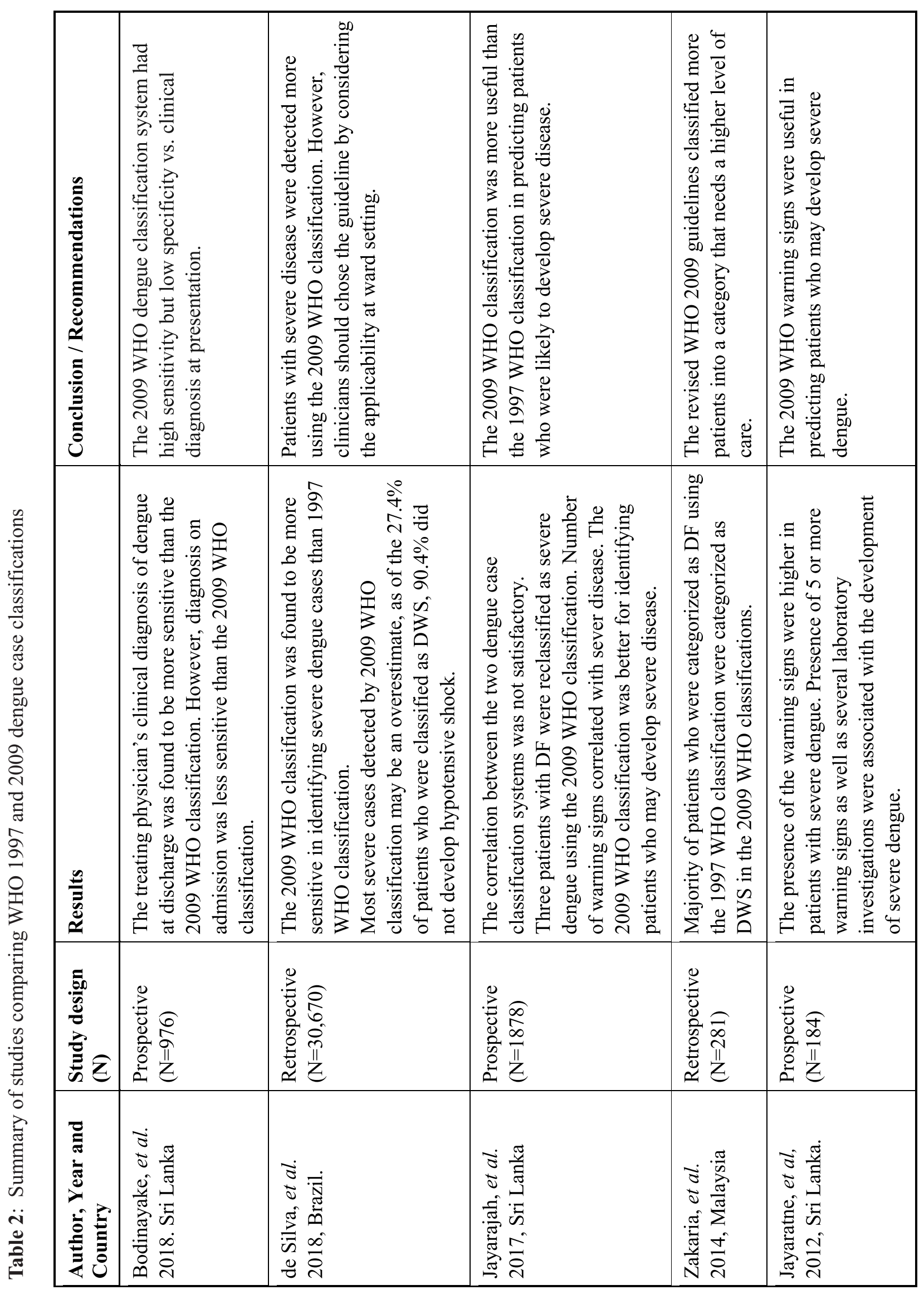




\begin{tabular}{|c|c|c|c|c|c|}
\hline 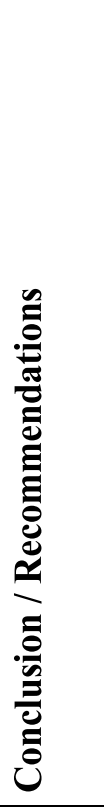 & 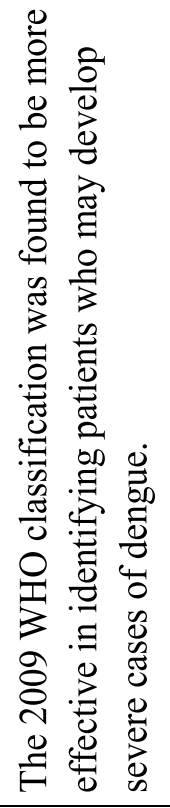 & 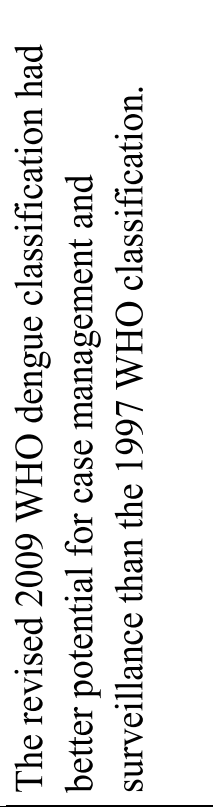 & 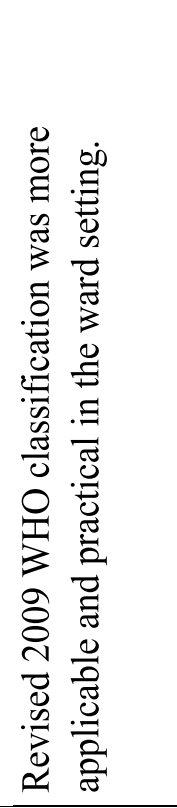 & 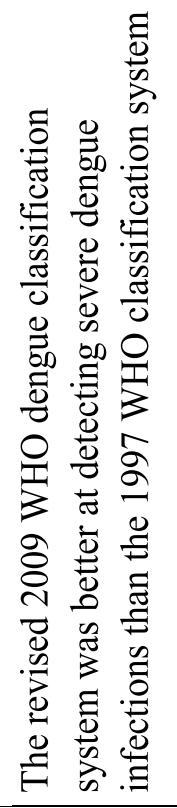 & 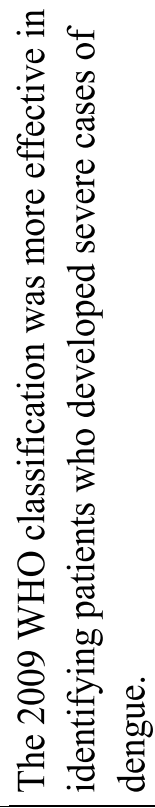 \\
\hline 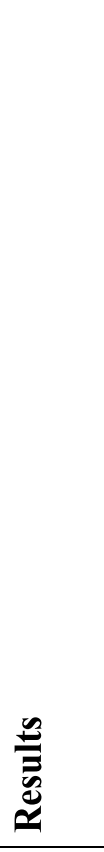 & 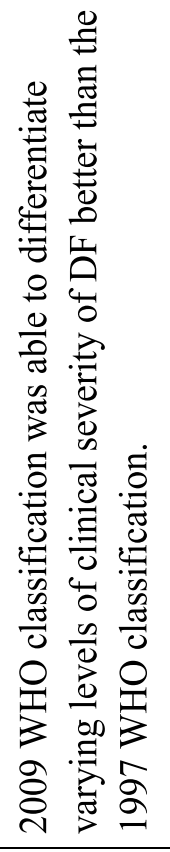 & 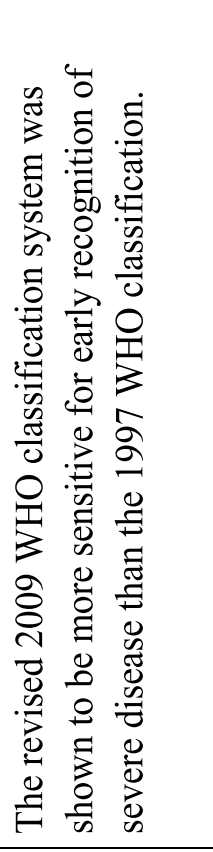 & 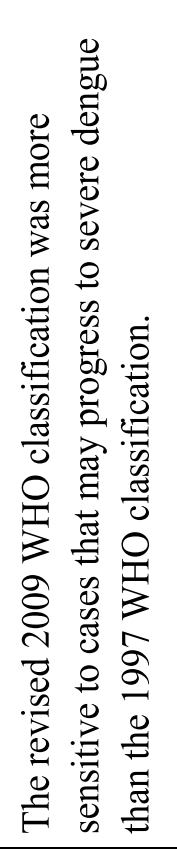 & 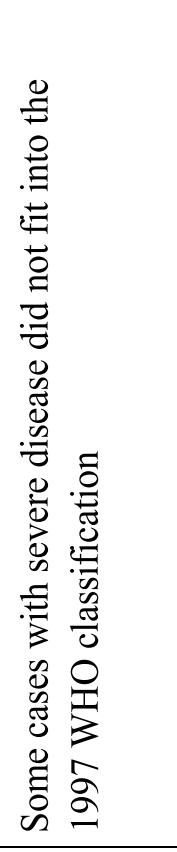 & 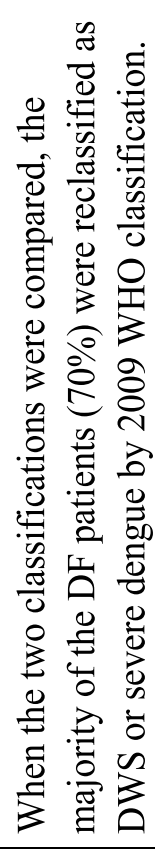 \\
\hline 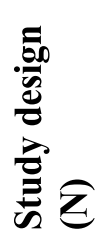 & 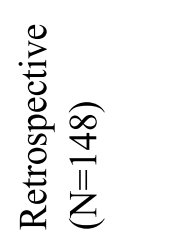 & 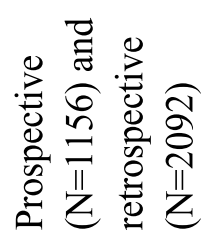 & 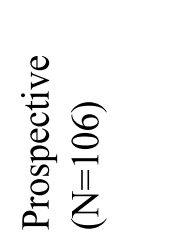 & 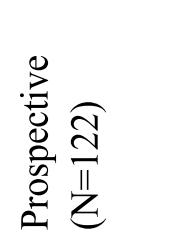 & 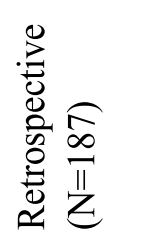 \\
\hline 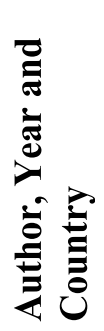 & 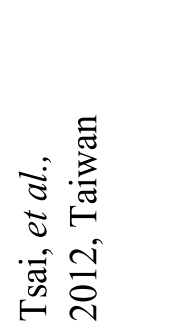 & 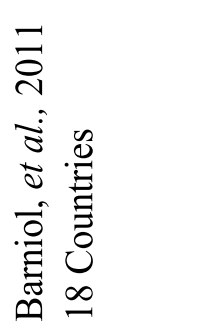 & 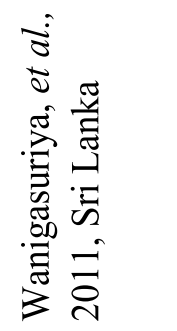 & 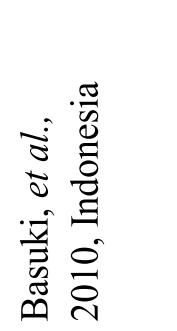 & 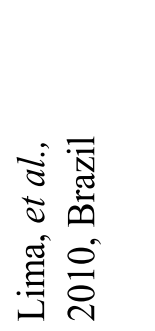 \\
\hline
\end{tabular}


DSS can be difficult to diagnose by inexperienced health care professionals, as patients may remain remarkably well in the early stages of shock and then deteriorate rapidly. In the 2009 WHO dengue classification, some criteria are not well-defined and may lead to differences in clinical assessment among physicians. For example, criteria such as: presence of severe bleeding and severe organ dysfunction are expected to be clinically judged by the treating physician. Thus, the diagnosis of severe dengue may vary among clinicians. This limitation should be overcome by more specific criteria for assessment of bleeding and organ dysfunction (9). Furthermore, in the 2009 classification "clinical" accumulation of fluid" is recognised as a warning sign. Clinical fluid accumulation occurs late and when fluid leak becomes clinically detectable, progression to impending shock may have already occurred. Therefore, it is not a useful warning sign in the clinical setting (22).

Furthermore, the 2009 classification may be difficult to practically incorporate into clinical practice in resource limited settings of developing nations due to the lack of rapid diagnostic tests and as most warning signs are not specific to dengue and may be seen in other tropical infections in such settings (9). In dengue endemic countries like Sri Lanka, all patients who present with fever and warning signs will invariably be diagnosed as having dengue with warning signs and warrant hospital admission for observation. This may increase in-hospital caseloads and in-turn put pressure on limited health budgets (9). Therefore, the 2009 classification needs revision to overcome these limitations and to be more clinically relevant.

Plasma leakage leading to haemodynamic instability with organ failure is a central pathophysiological feature of dengue. This needs to be detected early and promptly managed with fluid therapy to prevent complications. Massive bleeding and shock is more common in adults than children due to intake of NSAIDs, underlying peptic ulcers or due to late presentation (9). Furthermore, comorbidities such as diabetes mellitus, hypertension, ischaemic heart disease, chronic liver or kidney disease are more prevalent among adults and may complicate the clinical picture. Thus, in the WHO SEARO 2011 massive bleeding, co-morbidity and co-infections were were added as Expanded Dengue Syndrome (EDS) (Table 1) (9).

In conclusion, from studies done so far, the 2009 WHO dengue classification appears to be clinically more useful in detecting severe disease. However, the 2009 WHO dengue classification is still not the ideal classification for clinical use especially in resource limited developing countries. We have highlighted several limitations that should be addressed to make it more clinically relevant. Furthermore, any future dengue case classifications should cater to developing countries with limited health resources.

\section{Conflict of interest: None declared}

Acknowledgements: None

\section{References}

1. Gurugama P, Garg P, Perera J, Wijewickrama A, Seneviratne SL. Dengue viral infections. Indian Journal of Dermatology, 2010, 55(1): 68-78.

2. Jayarajah U, Faizer S, De Zoysa IM, Seneviratne SL. A large dengue epidemic affects Sri Lanka in 2017. Int J Prog Sci Technol, 2017, 6(1): 84-85.

3. Jayarajah U, Seneviratne SL, Gurugama P, Wanigasuriya $\mathrm{K}$ : Microalbuminuria and dengue viral infections. Southeast Asian J Trop Med Public Health, 2017; 48(4): 938.

4. Dissanayake HA, Seneviratne SL. Liver involvement in dengue viral infections. Reviews in Medical Virology, 2018: 28(2): e1971.

5. Gurugama P, Jayarajah U, Wanigasuriya K, Wijewickrama A, Perera J, Seneviratne SL. Renal manifestations of dengue virus infections. Journal of Clinical Virology, 2018, 101: 1-6.

6. Perera L, De Zoysa N, Jayarajah U, Senanayake N, De Zoysa I, Seneviratne SL. Transfusion-transmissible dengue infections. Transactions of The Royal Society of Tropical Medicine and Hygiene, 2020, 114 (In Press).

7. Jayarajah U, Basnayake O, Nagodavithane K, Jayasinghe J, Samarasekera DN. Atypical Presentation of Severe Dengue in a Patient following a Major Abdominal Surgery. Case Reports in Infectious Diseases, 2020; 2020. 
8. Horstick O, Jaenisch T, Martinez E, Kroeger A, See LLC, Farrar J, Ranzinger SR: Comparing the usefulness of the 1997 and 2009 WHO dengue case classification: a systematic literature review. The American Journal of Tropical Medicine and Hygiene, 2014; 91(3): 621-634.

9. Jayarajah U, Dissanayake U, Abeysuriya V, de Silva PK, Jayawardena P, Kulatunga A, Fernando H, Madarasinghe M, Hapugoda D, Perera L, et al., Comparing the 2009 and 1997 World Health Organization dengue case classifications in a large cohort of South Asian patients. The Journal of Infections in Developing Countries, 2020.

10. Ng D, Ghosh A, Jit M, Seneviratne SL. Mini-review: Can non-human leucocyte antigen genes determine susceptibility to severe dengue syndromes? Transactions of The Royal Society of Tropical Medicine and Hygiene, 2017; 111(9): 384-392.

11. da Silva NS, Undurraga EA, Verro AT, Nogueira ML. Comparison between the traditional (1997) and revised (2009) WHO classifications of dengue disease: a retrospective study of 30670 patients. Trop Med Int Health, 2018, 23(12): 1282-1293.

12. Lima FR, Croda MG, Muniz DA, Gomes IT, Soares KR, Cardoso MR, Tauro RL, Croda J. Evaluation of the traditional and revised World Health Organization classifications of dengue cases in Brazil. Clinics (Sao Paulo), 2013; 68(10): 1299-1304.

13. Tsai CY, Lee IK, Lee CH, Yang KD, Liu JW. Comparisons of dengue illness classified based on the 1997 and 2009 World Health Organization dengue classification schemes. J Microbiol Immunol Infect, 2013; 46(4): 271-281.

14. Basuki PS, Budiyanto, Puspitasari D, Husada D, Darmowandowo W, Ismoedijanto, Soegijanto S, Yamanaka A. Application of revised dengue classification criteria as a severity marker of dengue viral infection in Indonesia. Southeast Asian J Trop Med Public Health, 2010; 41(5): 1088-1094.

15. Zakaria Z, Zainordin NA, Sim BL, Zaid M, Haridan US, Aziz AT, Shueb RH, Mustafa M, Yusoff NK, Malik AS, et al., An evaluation of the World Health Organization's 1997 and 2009 dengue classifications in hospitalized dengue patients in Malaysia. J Infect Dev Ctries, 2014; 8(7): 869-875.
16. Barniol J, Gaczkowski R, Barbato EV, da Cunha RV, Salgado D, Martínez E, Segarra CS, Pleites Sandoval EB, Mishra A, Laksono IS, et al., Usefulness and applicability of the revised dengue case classification by disease: multicentre study in 18 countries. BMC Infectious Diseases, 2011; 11: 106.

17. de Silva P, Jayawardena P, Jayarajah U. Improving clinical outcomes through setting up of a specialised dengue treatment unit. Int JAdv Res, 2017; 5(11): 1152-1153.

18. Jayarajah U, de Silva PK, Jayawardana P, Dissanayake U, Kulatunga A, Fernando H, Perera L, Kannangara V, Udayangani C, Peiris R. Pattern of dengue virus infections in adult patients from Sri Lanka. Transactions of The Royal Society of Tropical Medicine and Hygiene, 2018; 112(3): 144-153.

19. Wanigasuriya K, Gurugama P, Wijewickrama A, Seneviratne S, Gunatilake S. Usefulness of World Health Organization (WHO) dengue case classifications in a Sri Lankan clinical setting. Journal of the Ceylon College of Physicians, 2012; 42(1-2).

20. Bodinayake CK, Tillekeratne LG, Nagahawatte A, Devasiri V, Arachchi WK, Strouse JJ, Sessions OM, Kurukulasooriya R, Uehara A, Howe S. Evaluation of the WHO 2009 classification for diagnosis of acute dengue in a large cohort of adults and children in Sri Lanka during a dengue-1 epidemic. PLoS Neglected Tropical Diseases, 2018; 12(2): e0006258.

21. Jayaratne S, Atukorale V, Gomes L, Chang T, Wijesinghe T, Fernando S, Ogg GS, Malavige GN. Evaluation of the WHO revised criteria for classification of clinical disease severity in acute adult dengue infection. BMC Research Notes, 2012; 5(1): 645.

22. Tissera H, Weeraman J, Amarasinghe A, Wijewickrama A, Palihawadana P, Fernando L. Expediency of dengue illness classification: The Sri Lankan perspective. WHO SouthEast Asia Journal of Public Health, 2014; 3(1): 5-7. 